\title{
THE
}

\section{Laser mimicking mosquito bites for skin delivery of malaria sporozoite vaccines}

\author{
Chang Zhou \\ Xinyuan Chen \\ University of Rhode Island, xchen14@uri.edu \\ Qi Zhang \\ Ji Wang \\ Mei X. Wu
}

Follow this and additional works at: https://digitalcommons.uri.edu/bps_facpubs

The University of Rhode Island Faculty have made this article openly available.

Please let us know how Open Access to this research benefits you.

This is a pre-publication author manuscript of the final, published article.

Terms of Use

This article is made available under the terms and conditions applicable towards Open Access Policy Articles, as set forth in our Terms of Use.

\section{Citation/Publisher Attribution}

Zhou, Chang, Xinyuan Chen, Qi Zhang, Ji Wang, and Mei X. Wu. "Laser mimicking mosquito bites for skin delivery of malaria sporozoite vaccines." Journal of Controlled Release, Vol. 204, 28 April 2015, Pages 30-37. Available: https://doi.org/10.1016/j.jconrel.2015.02.031

This Article is brought to you for free and open access by the Biomedical and Pharmaceutical Sciences at DigitalCommons@URI. It has been accepted for inclusion in Biomedical and Pharmaceutical Sciences Faculty Publications by an authorized administrator of DigitalCommons@URI. For more information, please contact digitalcommons-group@uri.edu. 


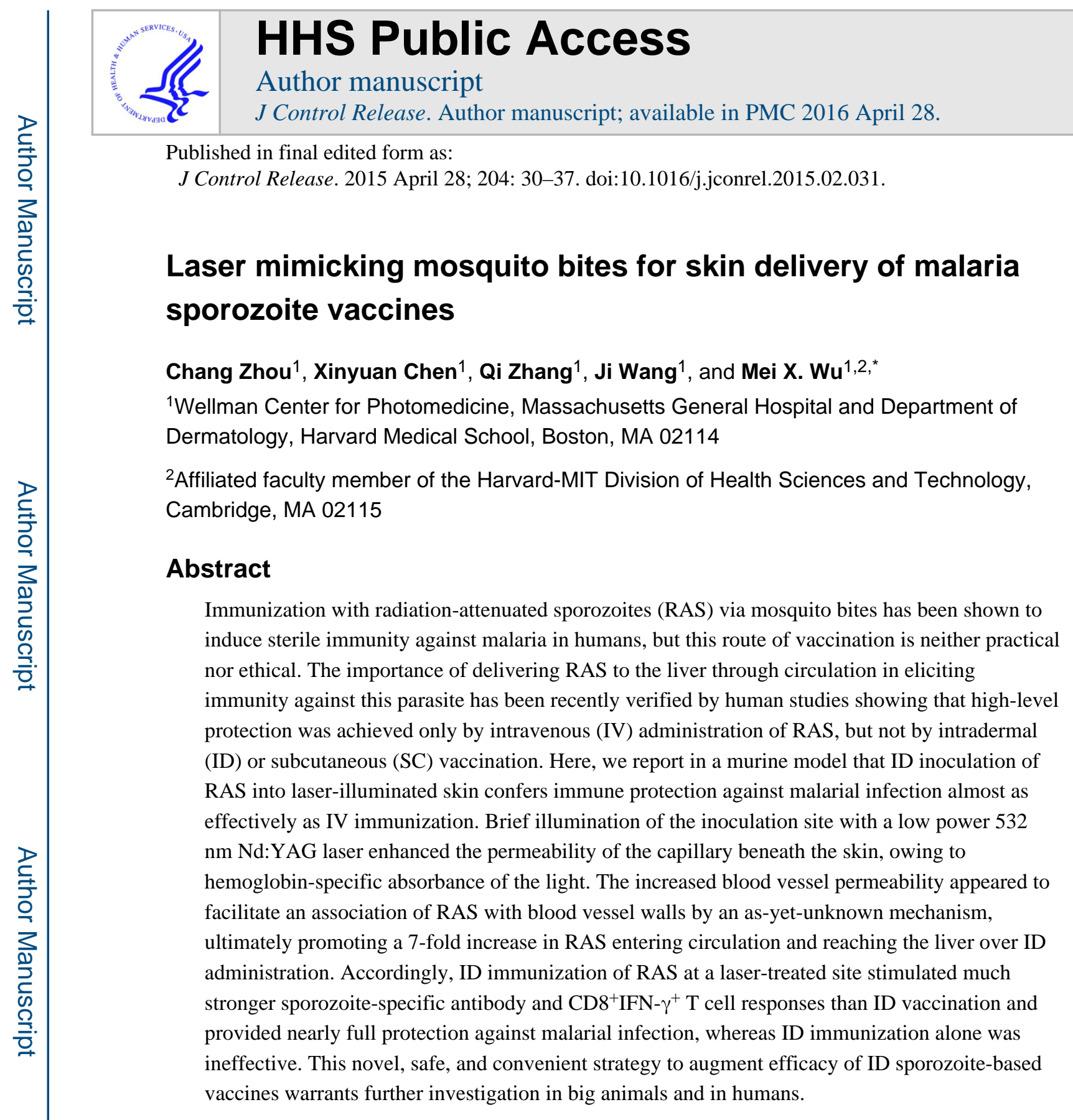

Keywords

Laser; malaria; sporozoites; vaccine; delivery

\footnotetext{
(C) 2015 Published by Elsevier B.V.

*Correspondence: Mei X. Wu, Wellman Center for Photomedicine, Edwards 222, Massachusetts General Hospital, 50 Blossom Street, Boston, MA 02114. Tel: (617)-726-1298; Fax: (617)-726-1206; mwu2@ partners.org.

Publisher's Disclaimer: This is a PDF file of an unedited manuscript that has been accepted for publication. As a service to our customers we are providing this early version of the manuscript. The manuscript will undergo copyediting, typesetting, and review of the resulting proof before it is published in its final citable form. Please note that during the production process errors may be discovered which could affect the content, and all legal disclaimers that apply to the journal pertain.
} 


\section{Introduction}

Malaria is a tropical disease mainly caused by plasmodium falciparum parasites and transmitted by infected mosquitoes. The WHO estimated that there were 207 million malarial infection cases and 627,000 related deaths in 2012 alone [1]. Effective vaccines would be the best strategy to control malarial epidemics if available, but currently the most advanced subunit malaria vaccine, RTS, S, can only provide about $50 \%$ protection in humans [2]. Another promising whole-parasite malaria vaccine, comprised of radiationattenuated plasmodium falciparum sporozoites (PfSPZ), confers $>80 \%$ protection in human volunteers [3]. All 6 volunteers who received 5 doses of intravenous (IV)-injected sporozoites and 6 out of 9 volunteers who received 4 doses were protected from malaria infection [3]. By traveling through circulation, radiation-attenuated sporozoites (RAS) reach the liver, infect hepatocytes, the only cells susceptible to malarial infection, and synthesize liver stage-specific antigens. These newly synthesized antigens, along with RAS, are major determinants for stimulating a protective immunity against malaria infection [4]. The ability of RAS to elicit immunity against sporozoites and liver-stage parasite partially explains why the whole-organism vaccine PfSPZ is more effective than RTS, S vaccine that consists of a single protein. Conceivably, for sporozoite-based vaccines, the greater amounts of RAS are loaded to the liver and the stronger the immunity could be induced. Although IV injection is an efficient route for delivering sporozoites to the liver, it faces formidable technical hurdles in vaccination of a large population. On the other hand, intradermal (ID) vaccination is a more clinically acceptable route and mimics natural infection. Yet, ID vaccination is far less efficient than IV immunization, probably because entrance of sporozoites into blood and lymphatic vessels is highly restricted in the dermis [5], especially for cryopreserved RAS that show reduced motility.

It remains unclear why RAS inoculated by mosquito bites can induce sterile immunity, whereas ID inoculation is less effective [6]. While it seemed plausible that mosquito bites directly inoculated RAS into blood vessels, resembling IV injections, recent studies found this was not the case because mosquitoes inoculated the majority of SPZs into the extravascular region of the dermis rather than directly into the vasculature [7-10]. The observation hardly explains the suboptimal immune responses and poor protection induced by ID or subcutaneous (SC) injection in clinical trials [11]. Similar results were also found in rodent models, in which immunization with cryopreserved RAS by IV injection provided 90-100\% protection, whereas immunizations via other routes such as ID, SC or intramuscular (IM) were far less effective [11-13] because fewer sporozoites reached the liver by these routes of immunization. To achieve a similar level of protection, a substantially higher number of sporozoites are required for ID or SC immunization [11, 13]. Unfortunately, dissection of sporozoites from infected mosquitoes is time-consuming, tedious, and cost-ineffective. Simply increasing the number of RAS per dose would increase the cost considerably, which would be problematic for a prophylactic vaccine needed by a large population in underdeveloped countries.

It is possible that ID injection of RAS by a syringe doesn't perform as mosquito bites, because when a mosquito probes blood, its proboscis damages the blood vessel. Sporozoites can then enter the blood vessel [14] in a process that may not be recapitulated by ID 
injection. For more than a decade, lasers have been used to treat vascular malformations in the clinics on the basis of "selective photothermolysis" [15]. Hemoglobin and oxyhemoglobin inside red blood cells have peak of light absorbance from $540 \mathrm{~nm}$ to $578 \mathrm{~nm}$ [16]. Thus, upon laser illumination near these wavelengths, abundant hemoglobin and oxyhemoglobin flowing inside blood vessels absorb laser energy and convert into heat to destroy malformed capillaries in human skin [17]. Since laser energy can be well controlled by energy density, laser power, pulse, and duration of illumination, we postulate that laser at a much lower energy density can slightly injure capillary vessels and promote entrance of sporozoites into blood vessels, simulating the process of mosquitoes' blood probing.

In the present study, different laser parameters are tested to selectively injure capillaries at the inoculation site. We found that a $532 \mathrm{~nm}$ laser at $1 \mathrm{~J} / \mathrm{cm}^{2}$ could increase permeability of the blood vessel and skin-to-liver delivery of ID-injected sporozoites significantly. Increased permeability of blood vessels appeared to facilitate an association of sporozoites with blood vessel walls via a yet unknown mechanism. The laser-mediated enhancement of skin-toliver delivery of RAS resulted in much stronger sporozoite-specific immune responses than ID vaccination alone and conferred protection against malarial infection nearly as effectively as IV immunization.

\section{Materials and Methods}

\section{Animals and parasites}

Female BALB/c mice at 8 weeks of age were purchased from Charles River Laboratory. Mosquitoes carrying Plasmodium yoelii-GFP (PyGFP) sporozoites were provided by Insectary Core Facility of Langone Medical Center, New York University. Fresh fluorescent sporozoites were isolated by dissecting mosquitoes under stereomicroscopy. Purified, aseptic, irradiated or non-irradiated $P$. yoelii sporozoites were preserved in the vapor phase of liquid nitrogen and provided by Sanaria (Maryland, USA). Animal protocols were approved by the Institutional Animal Care and Use Committee (IACUC) of Massachusetts General Hospital.

\section{Selective blood vessel injury by lasers}

A $532 \mathrm{~nm}$ Nd:YAG laser (Spectra-Physics Inc., Mountain View, CA) and a $595 \mathrm{~nm}$ pulsed dye laser (Vbeam, Candela, MA) were used in this study (Table 1). To test the effects of lasers on the permeability of capillaries beneath the skin, FITC-conjugated dextran (MW 200,000 ) was injected into the tail vein to label blood vessels of mice, and the dorsal skin was then illuminated by lasers at different settings. Leakage of FITC-dextran in laser-treated skin was examined by intravital confocal microscopy (Olympus). Alternatively, the skin was treated with laser, followed by FITC-dextran injection and microscopic analysis to determine duration of the leakage. For histological examination, laser-treated skin was collected immediately after laser treatment, fixed in $10 \%$ formalin, and processed by standard hematoxylin and eosin (H\&E) staining. Slides were analyzed by Nanozoomer Slide Scanner (Hamamatsu). 


\section{Confocal microscopy of ID-injected sporozoites}

Texas red-conjugated dextran (MW 70,000) was injected intravenously to label blood vessels. $P$. yoelii sporozoites were stained with carboxyfluorescein succinimidyl ester (CFSE) for $5 \mathrm{~min}$, washed twice by PBS, and resuspended in PBS. The stained sporozoites at a concentration of 5,000 sporozoites in $0.5 \mu \mathrm{PBS}$ were administered into mouse ears after illumination with $532 \mathrm{~nm}$ laser or sham light by a micro-liter syringe (Hamilton $1701 \mathrm{~N})$. The ears were harvested 15 minutes after the injection, fixed in $10 \%$ formalin, and examined under a confocal microscope (Olympus).

\section{Quantification of parasite loads in the liver}

To quantify parasite loads in the liver, mice were IV administered with varying numbers of sporozoites, and the liver was harvested $42 \mathrm{hr}$ later. Total RNA was extracted from an aliquot of the liver cells. Parasite-specific 18S rRNA was reverse transcribed and amplified by real-time PCR (the Roche SYBR Green system) or RT-qPCR with primers: forward, 5'GGGGATTGGTTTTGACGTTTTTGCG-3' and reverse, $5^{\prime}$ AAGCATTAAATAAAGCGAATACATCCTTAT- $3^{\prime}[18,19]$. The housekeeping gene $\beta$ actin was used as an internal control using primers: forward, $5^{\prime}$ -

CTGGGACGACATGGAGAAGATC-3' and reverse, 5'GTCTCAAACATGATCTGGGTCATC- $3^{\prime}$. The effect of laser on parasite loads in the liver was assessed by equivalency to the number of parasites in the liver after varying numbers of RAS were IV injected. For PyGFP sporozoites, equal amounts of freshly isolated PyGFP sporozoites were administered via IV or ID at laser-treated or sham-treated sites. Livers were harvested 42 hours later and dissociated to prepare single cell suspension by a $70 \mu \mathrm{m}$ cell strainer. Aliquots of liver cells were either extracted to obtain total RNA or analyzed by flow cytometry to count $\mathrm{GFP}^{+}$cells in the liver.

\section{Immunization and challenge}

Irradiated 2,000 P. yoelii sporozoites were either IV injected into the tail vein or ID injected into laser-treated or untreated dorsal skin. The immunization was repeated twice with 2week intervals. Mice were challenged by IV injection of 200 non-irradiated $P$. yoelii sporozoites 7 days after the last immunization. Blood samples were collected for thin blood smears analyzing percent parasitemia from days 3 to 21 post-challenge after Giemsa staining under a Zeiss Axiophot microscope.

\section{Immunofluorescence assay of sporozoite-specific antibody}

Sporozoite-specific antibody titer was measured by immunofluorescence assay 7 days after the final immunization as described [11]. Briefly, 2,000 sporozoites in $20 \mu \mathrm{l}$ PBS containing $2 \%$ bovine serum albumin (BSA) were coated to each well of Cel-Line slides (Thermo Scientific) and air dried. Pre-immune sera were diluted at 1:50, while immune sera were serially diluted starting from 1:50. The slide was incubated with $20 \mu$ diluted pre-immune and immune sera at $37^{\circ} \mathrm{C}$ for 1 hour in a moisture box. After washing three times in PBS, slides were incubated with FITC-conjugated rabbit anti-mouse IgG antibody for 1 hour. After wash, vectashield mounting medium (Vector laboratories) was added to each well before placing a cover glass to the slide. The slides were examined under an Olympus BX51 
fluorescence microscope at $400 \times$ magnification. Fluorescence intensity in each well was recorded and measured by ImageJ. The endpoint titer was defined as the highest dilution of immunized sera that had higher fluorescence intensity than the pre-immune sera.

\section{Measurement of sporozoite-specific T cell responses}

Mice were immunized three times each with 10, 000 sporozoites administered by IV or ID in the presence or absence of laser illumination as detailed above. The mice were sacrificed 7 days after the final immunization and the liver, spleen, and blood were collected. The liver and spleen were dissociated by a $70 \mu \mathrm{m}$ cell strainer. The cell suspensions, along with blood samples, were treated with Ammonium-Chloride-Potassium lysing buffer to remove red blood cells, and lymphocytes were isolated using Percoll (33\%) as described [20]. Lymphocytes were then stimulated with $1 \mathrm{mg} / \mathrm{ml}$ PyCSP280-288 peptide (SYVPSAEQI) for 21 hours at $37{ }^{\circ} \mathrm{C}$ with $1 \mu \mathrm{g} / \mathrm{ml}$ Golgin-plug in the culture for the final $5 \mathrm{hr}$. The stimulated cells were harvested, fixed with $2 \%$ formaldehyde, permeabilized with permeabilization buffer (eBioscience), and stained with indicated antibodies. Among the antibodies used, PerCP-Cy5.5 anti-Mouse CD8a antibody (clone 53-6.7) was purchased from eBioscience, PE anti-mouse CD11a Antibody (M17/4), Alexa 647 anti-mouse CD90.2 (30-H12) antibody, and FITC anti-mouse IFN- $\gamma$ antibody (XMG1.2) from Biolegend, antimouse CD16/CD32 antibody (2.4G2) from BD Biosciences. The stained cells were assessed on FACSAria (BD Biosciences) and analyzed by FlowJo software (version 7.6.5).

\section{Statistics}

One-way ANOVA followed by Tukey's multiple comparison tests were used to analyze the differences among multiple groups. Two-way ANOVA followed by Bonferroni post tests were used to analyze the parasitemia and compare the differences among selected groups. Log-rank test was used to analyze the survival data. All statistical analyses were performed using Prism GraphPad 6.

\section{Results}

\section{Laser illumination alters the permeability of capillaries in the skin}

To mimic mosquito bites, laser was used to target blood vessels in the skin. Previous studies have shown that lasers at $540 \mathrm{~nm}$ and $578 \mathrm{~nm}$ could induce selective blood vessel injury [15, 17]. We thus chose a $532 \mathrm{~nm}$ laser and a $595 \mathrm{~nm}$ laser for initial tests. Mice were IV injected with vessel-impermeable FITC-dextran to label the capillary network (Fig. 1A). After 532 $\mathrm{nm}$ laser treatment at a low dose of $1 \mathrm{~J} / \mathrm{cm}^{2}$, some dye leaked out and scattered over the laser-treated skin. Capillary vessels appeared to enlarge slightly compared with those in untreated skin (Figure 1A, panel 1 vs 2). Slight enlargement of capillary vessels was corroborated by histological examination and notably, there was no leakage of red blood cells out of the vessel in spite of the enlargement (Fig. 1C, arrow). The surrounding tissue of the dilated vessel was also normal (Figure 1B and C). As expected, the $532 \mathrm{~nm}$ laser at a higher dose of $9 \mathrm{~J} / \mathrm{cm}^{2}$ (total energy of $3.46 \mathrm{~J}$ ) led to more FITC-dextran leakage, concomitant with vessel injuries or rupture as evidenced by leakages of red blood cells from the vessel (Figure 1C). Treatment with the $595 \mathrm{~nm}$ laser at $5 \mathrm{~J} / \mathrm{cm}^{2}$ or $20 \mathrm{~J} / \mathrm{cm}^{2}$ resulted in robust leakage of FITC-dextran (Fig. 1A), as well as vessel rupture, respectively (Fig. 1C). 


\section{Laser promotes delivery of ID-injected sporozoites to the liver}

We next investigated whether the laser could promote delivery of ID-injected sporozoites to the liver. Since RAS did not replicate in the liver [21] and could not be detected by RTqPCR (data not shown), we used non-irradiated sporozoites instead. The amount of sporozoites that reached the liver after ID injection was calculated based on a standard curve generated by IV injection of varying numbers of sporozoites (Fig. 2A). As shown in Fig. 2B, ID injection of 4,000 sporozoites resulted in a parasite load that was equal to IV injection of about 400 sporozoites, suggesting that only $10 \%$ sporozoites entered blood circulation after ID inoculation. In contrast, illumination of the skin with $532 \mathrm{~nm}$ laser at $1 \mathrm{~J} / \mathrm{cm}^{2}$ gave rise to parasite liver loads equivalent to IV injection of about 2,800 sporozoites (Figure 2B), indicative of a 7-fold increase over ID inoculation alone. To our surprise, a high dose of laser at $9 \mathrm{~J} / \mathrm{cm}^{2}$ enhanced liver-delivery of sporozoites by only 4-fold. The less efficiency of laser at a higher dose hinted that severe blood vessel damage might reduce a skin-to-liver delivery of sporozoites. Although the underlying mechanism was unknown, a continuous flow of the bloodstream at the inoculation site might be crucial for sporozoites trafficking to the liver. Too much capillary damage by $595 \mathrm{~nm}$ laser may adversely affect traveling of sporozoites from the skin to the liver. Another possibility was that coagulation of red blood cells inhibited the motility of sporozoites as shown by previous investigation [10]. Consistent with this, the $595 \mathrm{~nm}$ laser did not enhance sporozoite delivery to the liver as effectively as $532 \mathrm{~nm}$ laser. Illumination with $595 \mathrm{~nm}$ laser for $5 \mathrm{~J} / \mathrm{cm}^{2}$ only increased the delivery by 2 -fold and no significant increase was seen with $20 \mathrm{~J} / \mathrm{cm}^{2}$. Accordingly, the $532 \mathrm{~nm}$ laser at $1 \mathrm{~J} / \mathrm{cm}^{2}$ was used in subsequent studies.

We next corroborated the effects of laser skin treatment on the trafficking of freshly isolated PyGFP sporozoites after ID administration [22] since cryopreserved sporozoites used in the preceding experiment might have a low vitality and infectivity. As shown in Fig. 2C, the percentage of $\mathrm{GFP}^{+}$cells in the liver was significantly higher in the presence of laser treatment than in the absence of the treatment. Liver parasite loads were also much higher in Laser+ID group than in ID group (Fig. 2D). These results suggest that laser could enhance skin-to-liver delivery of sporozoites whether the sporozoites are freshly isolated or cryopreserved.

To determine how laser treatment of the skin could facilitate delivery of ID-injected sporozoites to the liver, sporozoites were fluorescently labeled and injected into laser-treated or untreated skin, followed by confocal microscopic analysis. After ID injection, most sporozoites were randomly scattered in the skin, and only a few sporozoites were close to blood vessel walls in the absence of laser treatment (Fig. 3B). In contrast, many sporozoites bound tightly to blood vessel walls and some entered the vessels in laser-treated skin (Fig. 3C). The percentage of sporozoites that were associated with or inside blood vessels was significantly higher in Laser+ID group than in ID group (Fig. 3D). The observations suggest that laser treatment of the skin facilitated sporozoites moving towards and binding to blood vessels. Increased association of sporozoites with blood vessels was consistent with their efficient entrance of the bloodstream. 
Laser enhances the immune responses provoked by ID immunization of sporozoites

Laser-mediated enhancement in delivery of ID-injected sporozoites to the liver should translate into stronger immune responses. To verify this, the lower dorsal skin of mice was illuminated by laser, followed by ID inoculation of 10,000 RAS, while control mice received either IV or ID injection of an equal amount of RAS. After three immunizations, sporozoite-specific antibody titer was measured by immunofluorescence assays. As shown in Fig. 4A, anti-sporozoite antibody titer was substantially higher in laser+ID group than in the ID group, although it was lower than that in the IV group. Likewise, CD8 ${ }^{+} \mathrm{IFN}-\gamma^{+} \mathrm{T}$ cells in peripheral blood mononuclear cells (PBMCs) in Laser+ID group were significantly higher than those in ID group. The level of $\mathrm{CD} 8^{+} \mathrm{IFN}-\gamma^{+} \mathrm{T}$ cells was comparable to that in IV group (Fig. 4B).

It has been reported that protection against malarial infection depends on sporozoite-specific $\mathrm{CD}^{+} \mathrm{T}$ cells producing IFN- $\gamma$ in the liver [23-26]. When naive $\mathrm{CD} 8^{+} \mathrm{T}$ cells encountered sporozoites, the cells expressed a reduced level of CD8a while increasing CD11a on the cell surface $[27,28]$. Thus, percentages of $\mathrm{CD} 11 \mathrm{a}^{\text {hi }} \mathrm{CD} 8 \mathrm{a}^{\text {lo }}$ cells were analyzed in the liver and spleen and these cells were found to be significantly higher in the presence of laser treatment than in the absence of the treatment (Fig. 5A and 5B). Moreover, upon stimulation with a sporozoite T cell epitope peptide derived from the circumsporozoite protein (CSP280-288, SYVPSAEQI), significantly higher percentages of cells secreting IFN- $\gamma$ were attained in Laser+ID group than in the ID group and in both liver and spleen, confirming that Laser+ID immunization induced stronger immune responses than ID immunization alone (Fig. 5C).

\section{Laser plus ID immunization offers a high level of protection against malarial challenge}

A challenge study was next carried out to determine the efficacy of laser plus ID vaccination. Mice were immunized three times each with 2,000 RAS and challenged by IV injection of 200 infectious sporozoites 7 days after the last immunization. Blood parasitemia revealed that 7 out of 8 mice in Laser+ID group were protected, whereas all animals in ID group were infected (Table 2). The protection rate of Laser+ID group was $87.5 \%$, which was comparable to that of IV group, and significantly higher than the ID group $(\mathrm{p}<0.001)$. Percent parasitemia in Laser+ID group was significantly lower than that of ID group (Fig. 6A). This result was also verified by RT-qPCR analysis of blood parasite loads (Fig. 6B).

\section{Discussion}

Our present study shows that a brief illumination of the inoculation site by a low power laser followed by ID inoculation of RAS confers much stronger protection against malarial challenge than ID immunization alone. The protection was comparable to IV immunization and was associated with an increase in the number of irradiated sporozoites trafficking to the liver. In the absence of laser treatment, only $10 \%$ of sporozoites entered the blood vessels following ID injection (Fig. 2A), which was similar to previous studies showing that ID inoculation of $p$. yoelii sporozoites gave rise to liver parasite burden at a level 10- to 20-fold lower than IV inoculation [29]. This held truth for $p$. berghei-GFP-Luc sporozoites as well [30]. Accordingly, 7-10 times more sporozoites were required by ID immunization to achieve the same level of protection by IV vaccination [11]. Whereas the IV injection route 
is cumbersome, especially when it is performed in infants and young children, the high number of RAS required for effective ID immunization alone greatly increases the cost of the vaccine. In this regard, human studies showed that at least 5 doses of PfSPZ vaccine each at $1.35 \times 10^{5}$ sporozoites or a total of $6.75 \times 10^{5} \mathrm{PfSPZ}$ were needed to confer a full protection in a single person [3]. Considering that a six-person dissection team can dissect only 500 mosquitoes per hour and one mosquito contains $2 \times 10^{4}$ PfSPZ, it costs $4 \sim 5$ working hrs of a skilled technician to dissect a sufficient number of RAS for one vaccinee [31]. By illumination of a tiny area of the skin, the number of RAS for effective ID immunization can be reduced by 7 -fold in the basis of our investigation, leading to great cost savings. In the future, a small, handheld device can be fabricated incorporating laser illumination and a convenient ID injection device, like microneedles, for efficient and convenient delivery of RAS vaccines if the efficacy of laser-assisted ID immunization is confirmed in humans.

Amino et al. estimated that about $35 \%$ sporozoites inoculated by mosquitoes entered blood vessels in the skin [8], which was much higher than the $10 \%$ of cryopreserved sporozoites ID injected (Figure 2D). The low efficiency of ID-injected sporozoites across blood vessels may be caused by blunted motility of sporozoites after cryopreservation, as movement of these sporozoites was hardly detected under a confocal microscope in the skin. Vessel damages caused by mosquito bites may facilitate entrance of sporozoites into the bloodstream. To mimic mosquito bites, we used a laser to induce blood vessel injury and found that illumination of the skin with a $532 \mathrm{~nm}$ laser at $1 \mathrm{~J} / \mathrm{cm}^{2}$ enhanced vessel permeability without causing significant damages to the vessel structure. Increased blood vessel permeability, rather than severe blood vessel damage, was sufficient in facilitation of sporozoite travel from the skin to the liver. The efficiency was even greater than that of mosquito bites. Laser with a higher energy induced more severe vessel damages but failed to promote a skin-to-liver trafficking of sporozoites as efficiently as a low energy laser. Perhaps rupture of blood vessels and coagulation of red blood cells hindered the motility of the sporozoites, in agreement with an earlier study suggesting that the motility of sporozoites in hematoma-coagulation of red blood cells was greatly inhibited via an unknown mechanism [10]. Thus, it will be critical to avoid severe vessel rupture and hematomas with laser-assistance of ID immunization of sporozoites.

It remains unclear how sporozoites, after being inoculated into the skin, can navigate to capillary vessels and enter the bloodstream. One study suggests that sporozoites move randomly in the dermis until they happen to contact a blood vessel, then move along the vessel and penetrate across the vessel wall [8]. Under this assumption, sporozoites encountering a lymphatic vessel instead of a blood vessel would enter the lymphatic system $[8,32]$. Another possibility is that sporozoites are stimulated or guided toward a blood vessel. Vanderberg et al. demonstrated in vitro that serum albumin could significantly stimulate the motility of sporozoites [33, 34]. Similarly, Hellmann et al. postulated that environmental constraints directed the movement of sporozoites, although the nature of the environmental constraints remains uncharacterized [35]. Our current investigation suggests a random movement of sporozoites in the skin after ID administration and only a few sporozoites are located close to a blood vessel in the control skin (Fig. 3B). However, in 
laser-treated skin, substantially more sporozoites were found binding tightly to a vessel wall or inside a vessel (Fig. 3C). Because laser treatment enhances the permeability of blood vessels, it is possible that albumin leakage stimulated sporozoites to move toward and stick on a vessel wall in a manner similar to chemotaxis, although further investigation is required to conclude this.

$\mathrm{CD} 8{ }^{+} \mathrm{T}$ cells and IFN- $\gamma$ production in the liver are found to be essential for malarial protection [23-26]. Laser illumination followed by ID immunization induced a higher level of $\mathrm{CD} 11 \mathrm{a}^{\text {hi }} \mathrm{CD} 8 \mathrm{a}^{\text {lo }}$ cells secreting IFN- $\gamma$ than the ID group, in both liver and spleen (Fig. 5), in agreement with increased trafficking of sporozoites to the liver. This translated to protection against malaria challenge at a level comparable to IV immunization. Recent human studies clearly showed that a high level protection occurred only in volunteers IV but not ID or SC immunized [3,11]. The ability of laser to augment ID immunization almost as effectively as IV immunization warrants further investigation of the novel approach in big animals and humans.

\section{Acknowledgments}

We thank Dr. Richard R. Anderson for stimulating discussions, William Farinelli for suggestions and technical assistance in operation and maintenance of various laser machines, Jeffrey $\mathrm{H}$. Wu for editing, Jenny Zhao and Denny Cao in our Photopathology Core for helping with confocal microscopy and flow cytometry, and Sanaria company for purified sporozoites. This work was supported by the National Institutes of Health grants AI089779 and AI097696 to M.X.W.

\section{References}

1. World Health Organization. World Malaria Report 2013. 2013

2. Kester KE, Cummings JF, Ofori-Anyinam O, et al. Randomized, double-blind, phase 2a trial of falciparum malaria vaccines RTS, S/AS01B and RTS, S/AS02A in malaria-naive adults: safety, efficacy, and immunologic associates of protection. J Infect Dis. 2009; 200(3):337-46. [PubMed: 19569965]

3. Seder RA, Chang LJ, Enama ME, et al. Protection against malaria by intravenous immunization with a nonreplicating sporozoite vaccine. Science. 2013; 341(6152):1359-65. [PubMed: 23929949]

4. Hoffman SL, Goh LM, Luke TC, et al. Protection of humans against malaria by immunization with radiation-attenuated Plasmodium falciparum sporozoites. J Infect Dis. 2002; 185(8):1155-64. [PubMed: 11930326]

5. Conteh S, Chattopadhyay R, Anderson C, Hoffman SL. Plasmodium yoelii-infected A stephensi inefficiently transmit malaria compared to intravenous route. PLoS One. 2010; 5(1):e8947. [PubMed: 20126610]

6. Clyde DF, Most H, McCarthy VC, Vanderberg JP. Immunization of man against sporozite-induced falciparum malaria. Am J Med Sci. 1973; 266(3):169-77. [PubMed: 4583408]

7. Sidjanski S, Vanderberg JP. Delayed migration of Plasmodium sporozoites from the mosquito bite site to the blood. Am J Trop Med Hyg. 1997; 57(4):426-9. [PubMed: 9347958]

8. Amino R, Thiberge S, Martin B, et al. Quantitative imaging of Plasmodium transmission from mosquito to mammal. Nat Med. 2006; 12(2):220-4. [PubMed: 16429144]

9. Jin Y, Kebaier C, Vanderberg J. Direct microscopic quantification of dynamics of Plasmodium berghei sporozoite transmission from mosquitoes to mice. Infect Immun. 2007; 75(11):5532-9. [PubMed: 17785479]

10. Vanderberg JP, Frevert U. Intravital microscopy demonstrating antibody-mediated immobilisation of Plasmodium berghei sporozoites injected into skin by mosquitoes. Int J Parasitol. 2004; 34(9): 991-6. [PubMed: 15313126] 
11. Epstein JE, Tewari K, Lyke KE, et al. Live attenuated malaria vaccine designed to protect through hepatic CD8(+) T cell immunity. Science. 2011; 334(6055):475-80. [PubMed: 21903775]

12. Kramer LD, Vanderberg JP. Intramuscular immunization of mice with irradiated Plasmodium berghei sporozoites Enhancement of protection with albumin. Am J Trop Med Hyg. 1975; 24(6 Pt 1):913-6. [PubMed: 1106231]

13. Voza T, Kebaier C, Vanderberg JP. Intradermal immunization of mice with radiation-attenuated sporozoites of Plasmodium yoelii induces effective protective immunity. Malar J. 2010; 9:362. [PubMed: 21159170]

14. Kappe SH, Kaiser K, Matuschewski K. The Plasmodium sporozoite journey: a rite of passage. Trends Parasitol. 2003; 19(3):135-43. [PubMed: 12643997]

15. Anderson RR, Parrish JA. Selective photothermolysis: precise microsurgery by selective absorption of pulsed radiation. Science. 1983; 220(4596):524-7. [PubMed: 6836297]

16. Zijlstra WG, Buursma A, Meeuwsen-van der Roest WP. Absorption spectra of human fetal and adult oxyhemoglobin, de-oxyhemoglobin, carboxyhemoglobin, and methemoglobin. Clin Chem. 1991; 37(9):1633-8. [PubMed: 1716537]

17. Garden JM, Tan OT, Kerschmann R, et al. Effect of dye laser pulse duration on selective cutaneous vascular injury. J Invest Dermatol. 1986; 87(5):653-7. [PubMed: 3772159]

18. Hermsen CC, Telgt DS, Linders EH, et al. Detection of Plasmodium falciparum malaria parasites in vivo by real-time quantitative PCR. Mol Biochem Parasitol. 2001; 118(2):247-51. [PubMed: 11738714]

19. Bruna-Romero O, Hafalla JC, Gonzalez-Aseguinolaza G, Sano G, Tsuji M, Zavala F. Detection of malaria liver-stages in mice infected through the bite of a single Anopheles mosquito using a highly sensitive real-time PCR. Int J Parasitol. 2001; 31(13):1499-502. [PubMed: 11595237]

20. Doll KL, Butler NS, Harty JT. Tracking the total CD8 T cell response following whole Plasmodium vaccination. Methods Mol Biol. 2013; 923:493-504. [PubMed: 22990800]

21. Silvie O, Semblat JP, Franetich JF, Hannoun L, Eling W, Mazier D. Effects of irradiation on Plasmodium falciparum sporozoite hepatic development: implications for the design of preerythrocytic malaria vaccines. Parasite Immunol. 2002; 24(4):221-3. [PubMed: 12010486]

22. Ono T, Tadakuma T, Rodriguez A. Plasmodium yoelii yoelii 17XNL constitutively expressing GFP throughout the life cycle. Exp Parasitol. 2007; 115(3):310-3. [PubMed: 17049517]

23. Doolan DL, Hoffman SL. The complexity of protective immunity against liver-stage malaria. J Immunol. 2000; 165(3):1453-62. [PubMed: 10903750]

24. Doolan DL, Martinez-Alier N. Immune response to pre-erythrocytic stages of malaria parasites. Curr Mol Med. 2006; 6(2):169-85. [PubMed: 16515509]

25. Riley EM, Stewart VA. Immune mechanisms in malaria: new insights in vaccine development. Nat Med. 2013; 19(2):168-78. [PubMed: 23389617]

26. Weiss WR, Sedegah M, Beaudoin RL, Miller LH, Good MF. CD8+ T cells (cytotoxic/suppressors) are required for protection in mice immunized with malaria sporozoites. Proc Natl Acad Sci USA. 1988; 85(2):573-6. [PubMed: 2963334]

27. Rai D, Pham NL, Harty JT, Badovinac VP. Tracking the total CD8 T cell response to infection reveals substantial discordance in magnitude and kinetics between inbred and outbred hosts. $\mathrm{J}$ Immunol. 2009; 183(12):7672-81. [PubMed: 19933864]

28. Schmidt NW, Butler NS, Badovinac VP, Harty JT. Extreme CD8 T cell requirements for antimalarial liver-stage immunity following immunization with radiation attenuated sporozoites. PLoS Pathog. 2010; 6(7):e1000998. [PubMed: 20657824]

29. Inoue M, Culleton RL. The intradermal route for inoculation of sporozoites of rodent malaria parasites for immunological studies. Parasite Immunol. 2011; 33(2):137-42. [PubMed: 21226727]

30. Nganou-Makamdop K, Ploemen I, Behet M, et al. Reduced Plasmodium berghei sporozoite liver load associates with low protective efficacy after intradermal immunization. Parasite Immunol. 2012; 34(12):562-9. [PubMed: 23171040]

31. Hoffman SL, Billingsley PF, James E, et al. Development of a metabolically active, nonreplicating sporozoite vaccine to prevent Plasmodium falciparum malaria. Hum Vaccin. 2010; 6(1):97-106. [PubMed: 19946222] 
32. Yamauchi LM, Coppi A, Snounou G, Sinnis P. Plasmodium sporozoites trickle out of the injection site. Cell Microbiol. 2007; 9(5):1215-22. [PubMed: 17223931]

33. Kebaier C, Vanderberg JP. Initiation of Plasmodium sporozoite motility by albumin is associated with induction of intracellular signalling. Int J Parasitol. 2010; 40(1):25-33. [PubMed: 19654011]

34. Vanderberg JP. Studies on the motility of Plasmodium sporozoites. J Protozool. 1974; 21(4):52737. [PubMed: 4138523]

35. Hellmann JK, Munter S, Kudryashev M, et al. Environmental constraints guide migration of malaria parasites during transmission. PLoS Pathog. 2011; 7(6):e1002080. [PubMed: 21698220] 


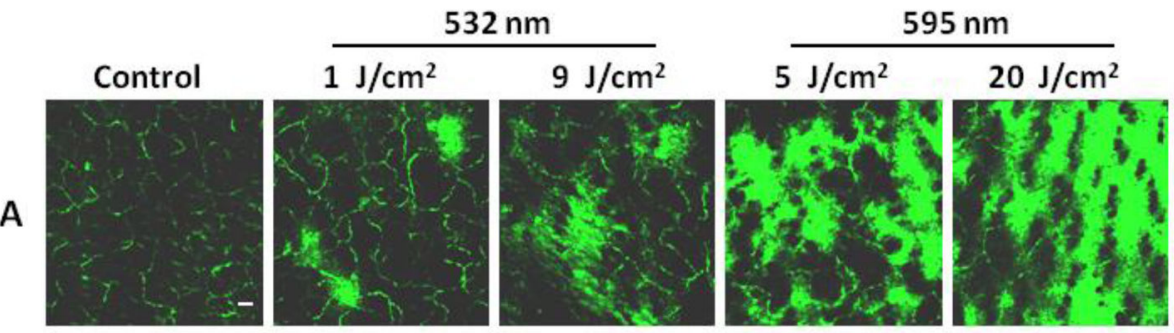

B
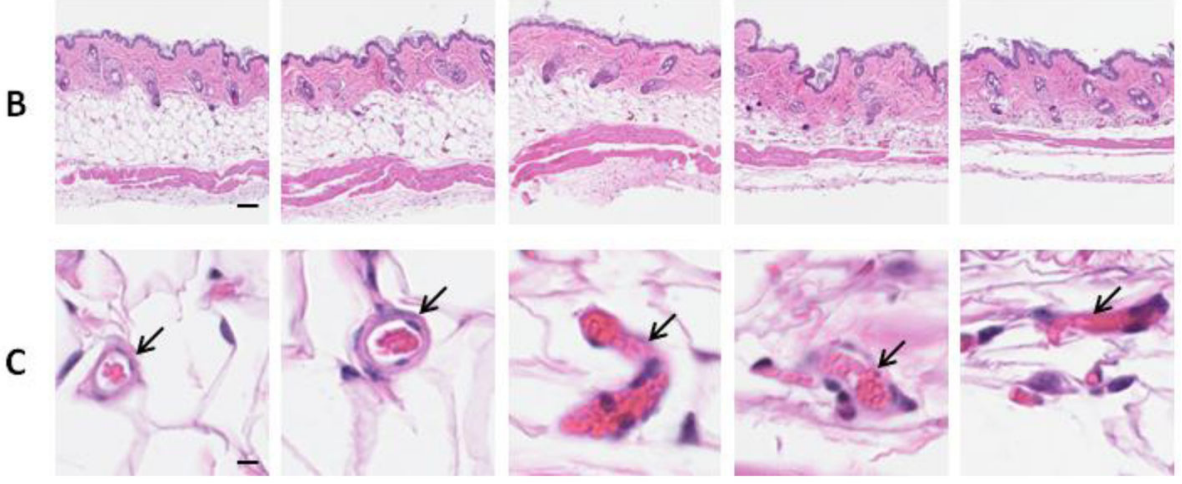

Figure 1.

Laser illumination enhances blood vessel permeability. (A) Representative images showing blood vessel leakage induced by laser. Blood vessels were marked by FITC-dextran IV injected, after which the dorsal skin of the mice was treated by different lasers and examined under an intravital confocal microscope within $30 \mathrm{~min}$. (B) Representative histological examination of laser-treated skins. (C) Alterations of blood vessels induced by lasers. Bar $=100 \mu \mathrm{m}$ in $A$ and B or $10 \mu \mathrm{m}$ in C. Arrows indicate blood vessels. $\mathrm{n}=5$. 
A

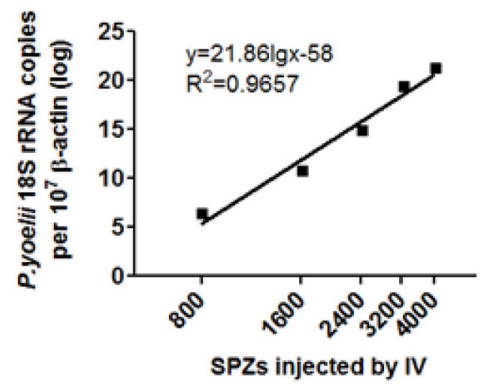

C

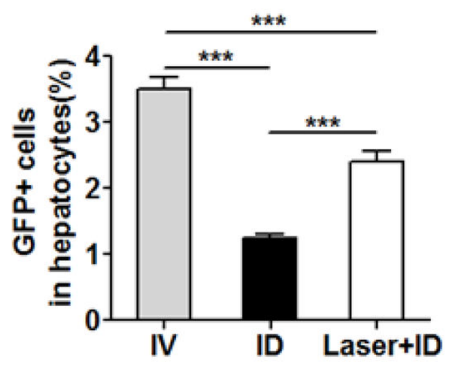

B

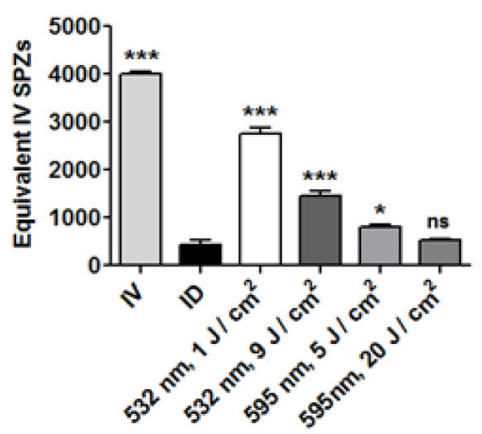

D

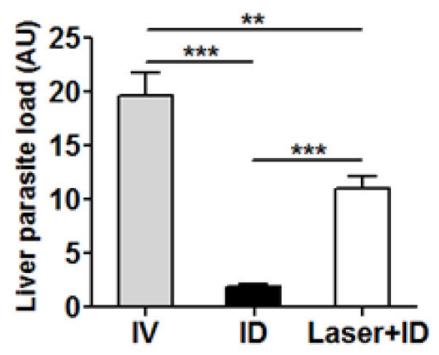

Figure 2.

Laser enhances the delivery of sporozoites from the skin to liver. (A) A standard curve of liver parasite loads after IV injection of indicated numbers of sporozoites. (B) Effects of laser skin illumination on skin-to-liver delivery of sporozoites. Mice were injected with 4,000 sporozoites either by an IV route or through ID injection into laser-treated or untreated dorsal skin (ID). The liver parasite loads were determined by RT-qPCR and estimated using the standard curve $2 \mathrm{~A}$. (C and D). $\mathrm{GFP}^{+}$cells were measured by flow cytometry $42 \mathrm{hrs}$ after freshly isolated PyGFP sporozoites were injected by IV or ID in the presence or absence of laser treatment (C). Liver parasite loads were quantified by RTqPCR in the animals (D). Results are expressed as means \pm standard deviation (SD). The experiment was repeated twice with similar results. $\mathrm{n}=8,{ }^{*} \mathrm{p}<0.05, * * \mathrm{p}<0.01, * * * \mathrm{p}<0.001$, and ns, not significant. 

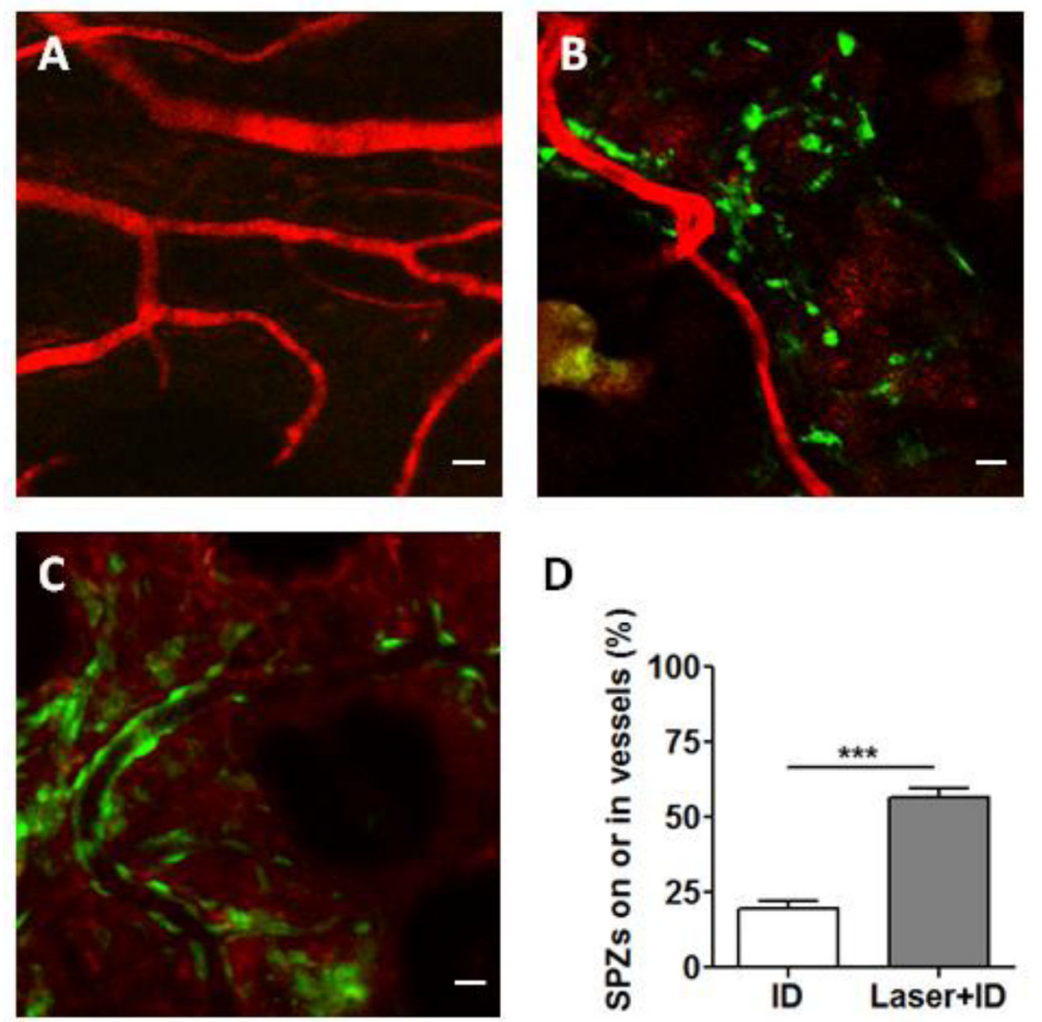

D

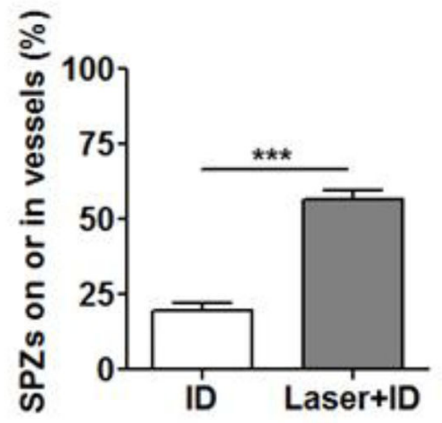

Figure 3.

Confocal microscopy of sporozoites in the skin. (A) Blood vessels were marked by Texas red-dextran (MW 70,000). (B and C) Representative images of CFSE-stained sporozoites in un-treated (B) or laser-treated skin (C). Bar $=10 \mu \mathrm{m}$. (D) Percentages of sporozoites in association with vessel walls or inside the vessels. Data are shown as means \pm SD. $n=10$, $* * * \mathrm{p}<0.001$. 

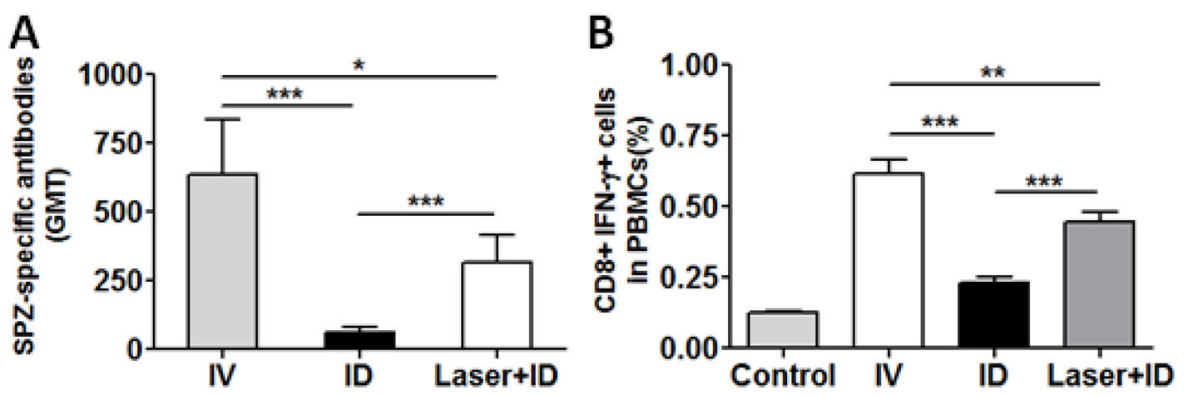

Figure 4.

Peripheral immune responses against sporozoites. Mice were immunized with three doses of RAS each with 10,000 sporozoites at an interval of two weeks. Geometric mean titers (GMT) of anti-sporozoite antibody were determined by immunofluorescence assay (A) and $\mathrm{CD}^{+} \mathrm{T}$ cells in PBMCs were analyzed by flow cytometry (B) 7 days after the final immunization. The results are expressed as means \pm SD. $N=8, * p<0.05$, ** $p<0.01$, and $* * * \mathrm{p}<0.001$. 
A

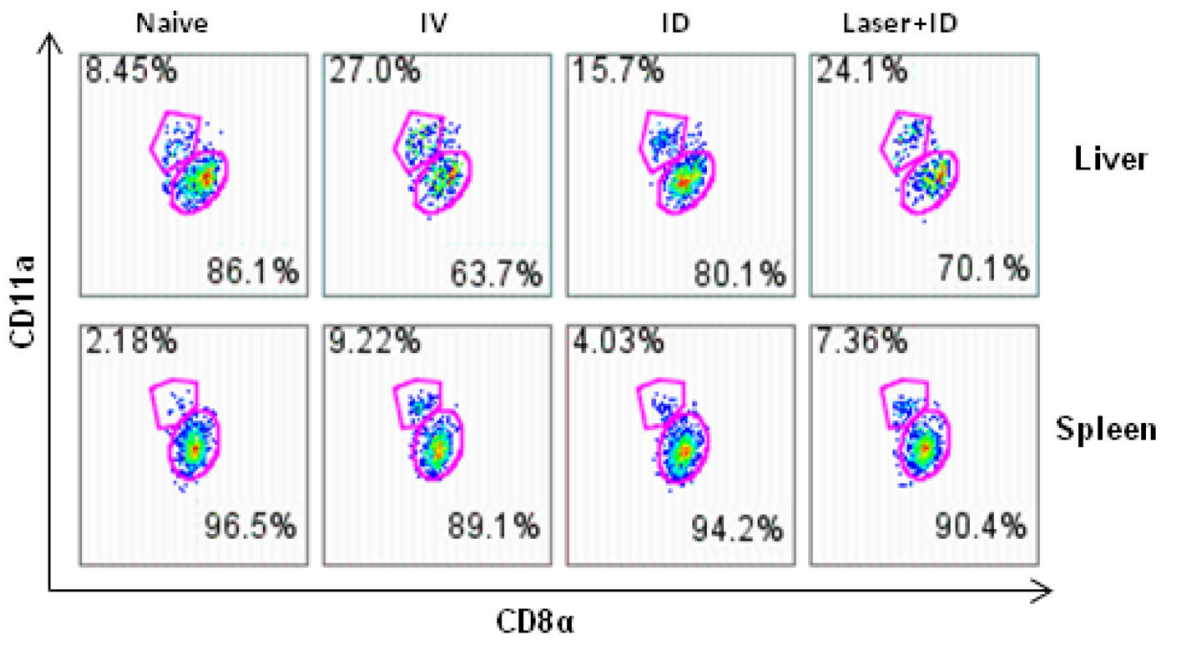

B

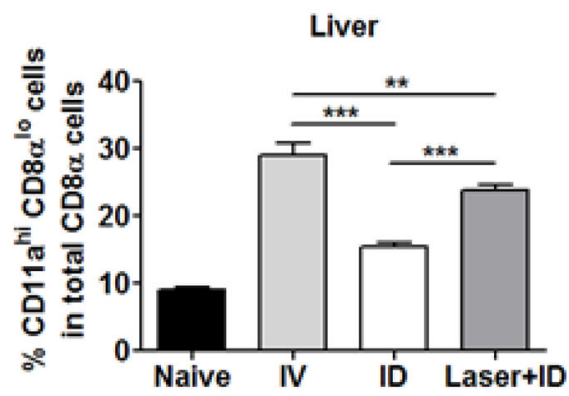

C

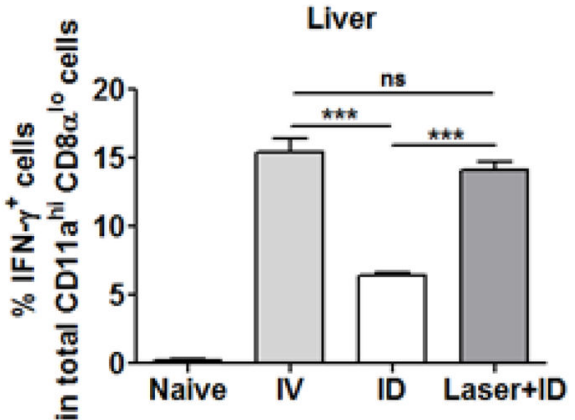

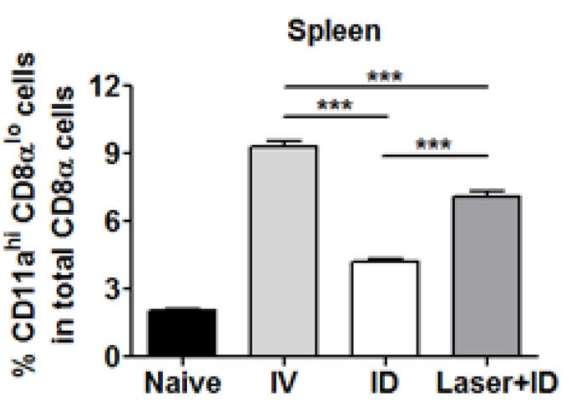

Spleen

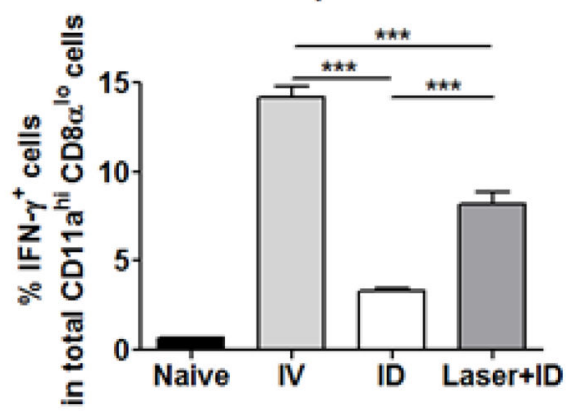

Figure 5.

Frequencies of sporozoite-specific $\mathrm{CD}^{+} \mathrm{T}$ cells in the liver and spleen. Mice were immunized with three doses of RAS each with 10,000 sporozoites at an interval of two weeks. Representative flow profiles of sporozoite-experienced CD $11 \mathrm{a}^{\text {hi }} \mathrm{CD} 8 \mathrm{a}^{\text {lo }}$ cells in the liver and spleen are shown in (A). Mean frequencies \pm SD of CD11a hi CD8 $a^{\text {lo }}$ cells (B) and IFN- $\gamma^{+}$-producing $\mathrm{CD}^{+} \mathrm{T}$ cells $(\mathrm{C})$ were attained in the liver and spleen by flow cytometry 7 days after the final immunization. $\mathrm{n}=8, * * \mathrm{p}<0.01, * * * \mathrm{p}<0.001$ and $\mathrm{ns}$, not significant. 

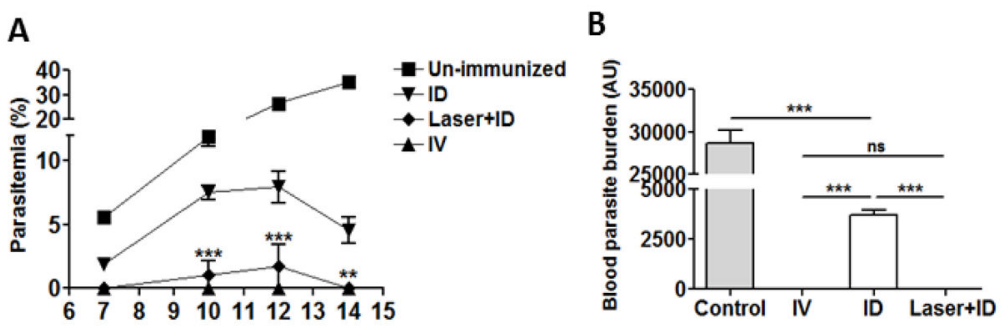

Figure 6.

Protection against malarial challenge. Mice were immunized with three doses of RAS each with 2,000 sporozoites at an interval of two weeks. All animals were challenged by IV injection of 200 live $P$. yoelii sporozoites 7 days after the final immunization. Parasitemia were monitored at indicated days post-challenge by blood smear (A) and blood parasite burdens were determined by RT-qPCR on day 10 post challenge (B). The results are expressed as Mean \pm SD. $n=8,{ }^{* *} \mathrm{p}<0.01$ and ${ }^{* * *} \mathrm{p}<0.001$ between ID and Laser+ID group in A or ***p $<0.001$ and ns, not significant in B. 


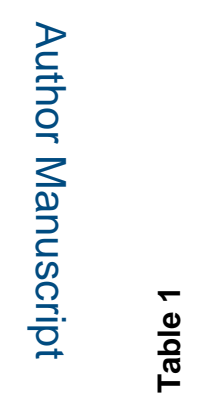

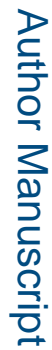

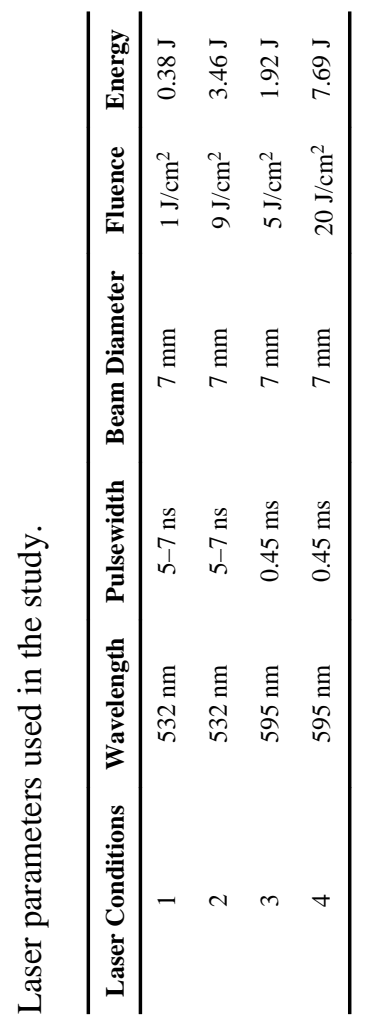

J Control Release. Author manuscript; available in PMC 2016 April 28. 


\section{Table 2}

Result of the challenge study.

\begin{tabular}{ccc}
\hline Immunization route & Infected/Injected & Protection \\
\hline Un-immunized & $8 / 8$ & 0 \\
IV & $0 / 8$ & $100 \%$ \\
ID & $8 / 8$ & 0 \\
Laser+ID & $1 / 8$ & $87.5 \%^{* * *}$ \\
\hline$* * *$ \\
$\mathrm{p}<0.001$ compared with ID group.
\end{tabular}

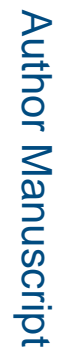

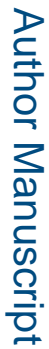

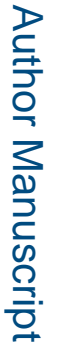

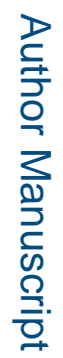

